\title{
Assistência de enfermagem a um paciente em convalescência por pênfigo: um relato de caso
}

Nursing care to a patient in convalescence by pephigh: a case report

Cuidados de enfermería a un paciente en convalecencia por pephigh: reporte de un caso

\section{Resumo}

Introdução: $\bigcirc$ pênfigo é uma doenças relativamente rara caracterizada pela formação de bolhas na pele e, às vezes, também nas mucosas (como boca, garganta, olhos, nariz e região genital de homens e mulheres). Objetivo: descrever um estudo de caso sobre uma paciente com pênfigo vulgar internada em um hospital referência em doenças tropicais do Amazonas. Métodos: pesquisa retrospectiva, descritiva com abordagem quantitativa. Resultados: Paciente há 10 dias iniciou quadro de bolhas finas que rapidamente evoluíram para erosões, com crostas hemáticas em região de face, dorso, tórax, abdome e membros inferiores (coxas e pernas). Também apresentando lesões bolhosas exsudativas, sangrantes aderentes as roupas de cama, disseminadas em todo o corpo, sem sinais de infecção, lesões de categoria dois. Foi evidenciado sinais de baixa-alto-estima, chorosa, poliqueixosa, expressão facial de angustia. Conclusão: há uma grande necessidade de padronizações em cuidados relacionados as lesões de pele, principalmente as que exigem complexidade em seu manejo como os pacientes portadores de pênfigo vulgar e que o paciente deve ser assistido como um todo.

Descritores: pênfigo, doenças raras, dermatopatia, estudo de caso único.

\section{Abstract}

Introduction: Pemphigus is a relatively rare disease characterized by the formation of blisters on the skin and sometimes also on the mucous membranes (such as the mouth, throat, eyes, nose and genital region of men and women). Objective: to describe a case study of a patient with pemphigus vulgaris admitted to a reference hospital for tropical diseases in the Amazon. Methods: retrospective, descriptive research with a quantitative approach. Results: For 10 days, the patient started to have fine blisters that quickly evolved to erosions, with hematic crusts on the face, back, chest, abdomen and lower limbs (thighs and legs). Also showing exudative blistering lesions, bleeding adhering to bed linen, disseminated throughout the body,
Kalíssia Moraes de Araújo

Enfermeira especialista na Graduanda de Enfermagem do Centro Universitário Nilton Lins - UNINILTONLINS: ORCID: 0000-0002-9145-5777

\section{Thaís Monteiro de Araújo}

Graduanda de Enfermagem do Centro

Universitário Nilton Lins - UNINILTONLINS:

ORCID: 0000-0003-2386-3813

\section{Elaine Fabrícia Oliveira da Silva}

Enfermeira especialista assistencial na Fundação de Medicina Tropical Dr. Heitor Vieira Dourado-FMT/HVD, ORCID: 0000-0002-5 11 12-71 11

\section{Ana Vitória Bezerra Costa}

Graduanda de Enfermagem do Centro Universitário Nilton Lins - UNINILTONLINS

ORCID: 0000-000 1-8548-3141

Izabela Cristina da Silva dos Santos Enfermeira especialista assistencial na Fundação de Medicina Tropical Dr. Heitor Vieira Dourado-FMT/HVD, ORCID: 0000-0002-33 1 1-5571 
without signs of infection, category two lesions. Signs of low self-esteem, tearful, multi-complaining, and distressed facial expression were evidenced. Conclusion: there is a great need for standardization of care related to skin lesions, especially those that require complexity in their management, such as patients with pemphigus vulgaris and that the patient must be assisted as a whole.

Descriptors: pemphigus, rare diseases, dermatopathy, single case study

\section{Resumen}

Introducción: El pénfigo es una enfermedad relativamente rara caracterizada por la formación de ampollas en la piel y en ocasiones también en las membranas mucosas (como boca, garganta, ojos, nariz y región genital de hombres y mujeres). Objetivo: describir un estudio de caso de un paciente con pénfigo vulgar ingresado en un hospital de referencia por enfermedades tropicales en la Amazonía. Métodos: investigación descriptiva retrospectiva con enfoque cuantitativo. Resultados: Durante 10 días la paciente comenzó a presentar ampollas finas que rápidamente evolucionaron a erosiones, con costras hemáticas en cara, espalda, tórax, abdomen y miembros inferiores (muslos y piernas). También presenta lesiones ampollosas exudativas, sangrantes adheridas a la ropa de cama, diseminadas por todo el cuerpo, sin signos de infección, lesiones de categoría dos. Se evidenciaron signos de baja autoestima, expresión facial Ilorosa, con múltiples quejas y angustia. Conclusión: existe una gran necesidad de estandarización de la atención relacionada con las lesiones cutáneas, especialmente aquellas que requieren complejidad en su manejo, como los pacientes con pénfigo vulgar y que el paciente debe ser atendido en su totalidad.

Descriptores: pénfigo, enfermedades raras, dermatopatía, estudio de caso único.

\section{Deisi Laís Muller}

Nutricionista especialista, assistencial na da Fundação de Medicina Tropical Dr. Heitor Vieira Dourado-FMT/HVD. ORCID: 0000-0002-3410-7215

\section{Kellyanne Trajano Alves}

Graduanda de Enfermagem do Centro Universitário Nilton Lins - UNINILTONLNS:

ORCID: 0000-0003-2084-9303

\section{Weverson Menezes de Azevedo}

Enfermeiro especialista, assistencial na Fundação de Medicina Tropical Dr. Heitor Vieira Dourado-FMT/HVD, ORCID: 0000-0001-8541-0292

\section{Arimatéia Portela de Azevedo} Enfermeiro Mestre - Coordenador da Comissão de Controle de Infecção Hospitalar $(\mathrm{CClH})$ e Comissão de Feridas da Fundação de Medicina Tropical Dr. Heitor Vieira DouradoFMT/ HVD

ORCID: 0000-0002-5434-4656

RECEBIDO 16/07/2021 | APROVADO: 17/10/2021

\section{INTRODUCÃO}

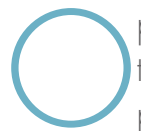
pênfigo, é uma doença autoimune, considerada uma patologia rara. Atinge de 0,5 a 3,2 pessoas por ano no mundo. Esta patologia está associada a fatores genéticos, imunológicos e ambientais, afeta com maior incidência os judeus e japoneses ${ }^{1}$

Existem dois tipos clínicos mais frequentes de pênfigo, o vulgar e o foliáceo. No pênfigo vulgar, a acantólise ocorre na camada basal, podendo acometer pele e mucosas, considerada a forma clinicamente mais grave, cuja incidência mundial é de 0,75$5 / 1.000 .000$ casos ao ano. No pênfigo foliáceo, a acantólise ocorre abaixo da camada córnea, ou seja, mais superficial, raramente acometendo a mucosa. A variante vulgar é relativamente rara, ocorre mais frequentemente após os 40 anos, acometendo ambos os sexos?

$\bigcirc$ pênfigo vulgar (PV) é a forma mais grave da doença, neste caso os pacientes apresentam bolhas em mucosas de diferentes órgãos e regiões do organismo. Já o pênfigo foliáceo (PF), também chamado de fogo selvagem (FS), tem como característica principal o aparecimento de bolhas na pele, decorrentes da acantólise, que se caracteriza pela separação das células da epiderme que ao se romperem dão origem a lesões dolorosas com odor fétido, deixando o indivíduo predisposto a infecções ${ }^{4}$. 
elemento semiotécnico para um diagnóstico mais simples do pênfigo vulgar é através do exame clínico com a verificação do sinal de Nikolsky. Esse sinal é considerado positivo quando, ao se friccionar fortemente a pele ou mucosa do paciente portador de pênfigo com a polpa digital ou com um instrumento rombo, ocorre o deslocamento do epitélio e surge no local uma superfície úmida tendendo para a cor rosada ou uma bolha ${ }^{1,3,5}$.

tratamento convencional é baseado no uso de medicamentos corticoides, mas existem relatos da utilização de imunoglobulina, com resultados positivos $^{2,8}$.

Também é utilizada uma estratégia paliativa com medicamentos imunomodulares tópicos e sistémicos para controlar a formação de bolhas e ajudar na cicatrização da mucosa ${ }^{6}$.

$\bigcirc$ pênfigo foliáceo possui as mesmas características epidemiológicas do vulgar em todas as partes do mundo, entretanto, no Brasil possui aspectos epidemiológicos endêmicos distintos, com elevada frequência na zona rural de alguns estados brasileiros. E possui provável conotação genética, com frequência familiar de 12\% dos $\operatorname{casos}^{9,10,12}$

pênfigo, enquanto fenômeno epidemiológico, não possui a mesma expressão populacional que a diabetes, doenças cardiovasculares, entre outras, mas temos experiências e vivências que precisam ser atendidas nesta realidade de vida, e estas práticas precisam ganhar contornos de intervenção em todas as esferas de assistência em saúde" .

Essa dermatose é caracterizada pelo aparecimento de bolhas frágeis que, ao se romperem, expõem ulcerações; as bolhas podem ocorrer nas diferentes camadas da epiderme, o que definirá qual camada será acometida e qual será o tipo de pênfigo, pois o mesmo possui cinco variações: pênfigo vulgar, pênfigo vegetante, pênfigo foliáceo, pênfigo eritematoso e pênfigo paraneoplásico $7,8,13$.

A fisiopatologia do PV inclui a perda
O pênfigo foliáceo possui as mesmas características epidemiológicas do vulgar em todas as partes do mundo, entretanto, no Brasil possui aspectos epidemiológicos endêmicos distintos, com elevada frequência na zona rural de alguns estados brasileiros. E possui provável conotação genética, com frequência familiar de $12 \%$ dos casos do contato célula-célula (acantólise) no epitélio, levando a formação de boIhas intra-epiteliais. Isso é, decorrente de dano desmossomo - estruturas responsáveis pela aderência das células às estruturas adjacentes e ao reconhecimento e sinalização célula-célula, provocados por autoanticorpos ${ }^{14}$.

A etiologia do PV é desconhecida, porém a patogenia envolve a produção anormal, de auto anticorpos que se fixam em estruturas da adesão da superfície das células epiteliais, que acabam por agir como antígenos. Esses supostos antígenos são glicoproteínas transmembrânicas que fazem parte da estrutura do desmossomo responsável pela união celular intra-epitelial - e são conhecidas como desmogleínas e desmocolinas ${ }^{15}$.

O PV encontra-se relacionado às desmogleínas, sendo que, dependendo do tipo de desmogleína envolvida na patologia, há variação quanto à localização das lesões. Quando a atuação dos autoanticorpos age contra apenas as desmogleínas tipo 3, há formação de bolhas na mucosa; o acometimento mucocutâneo tem relação com as desmogleínas dos tipos 1 e 3. Os auto-anticorpos se unem a esses componentes desmossomais, por conseguinte inibem de maneira efetiva a interação molecular que é responsável pela aderência ${ }^{16}$.

A síntese de anticorpos lgG4 Dsg 1 afeta a desmogleína 1 presente na derme e anticorpos lgG4 Dsg3 acometem a desmogleína 3 existente na mucosa; por conseguinte, as bolhas intraepiteliais são resultados da acantólise, ou seja, da perda de adesão 
dos queratinócitos, pois essa adesão se deve às desmogleínas 1 e 3 que são componentes dos desmossomos, cuja função é fazer a conexão das células epiteliais ${ }^{17,18}$.

A maior parte dos indivíduos acometidos são hiponutrido e habitantes da zona rural. Os primeiros sinais são manchas eritematosas na pele, desenvolvendo-se a seguir nessas regiões bolhas flácidas que se rompem ao menor toque, quando as infecções secundárias se instalam. As porções inicialmente atingidas são a face e o tórax, progredindo depois em sentido craniocaudal. $\bigcirc$ vegetante é uma variante do pênfigo vulgar, sendo que as bolhas dão lugar a massas verrucóides vegetantes. Após a formação das bolhas desenvolve-se tecido de granulação hipertrófico nas áreas desnudadas ${ }^{19}$.

As ulcerações bucais são mais raras que no pênfigo vulgar, dando lugar a crescimentos friáveis que sangram ao menor contato. Apesar de ser uma doença predominantemente cutânea, o pênfigo vegetante, no início, envolve o vermelhão dos lábios e a mucosa bucal. $\bigcirc$ eritematoso é considerado uma forma frustra de pênfigo foliáceo, sendo representada por bolhas ou eritemas que atingem a face, região pré-esternal e média dorsal. Com evolução lenta, mínima sintomatologia e sem comprometimento do estado geral do paciente ${ }^{20,21}$.

As feridas por pênfigo são disseminadas, irritantes com a sensação de ardor, às vezes são pruriginosas, formas bolhas e feridas com tempo de cicatrização prolongado, provavelmente, têm infecção sobreposta e quando associadas à idade avançada ampliam a gravidade. Essas condições ainda podem ser mais acentuadas se forem associados a comorbidades pré-existentes como as doenças crôni- co-degenerativas $5,8,19$.

Histologicamente, caracteriza por uma separação intra-epitelial (vesícula ou bolha intra-epitelial) acima da camada basal das células do epitélio, sendo que esta permanece aderida à lâmina própria subjacente. Algumas vezes, toda a camada superficial do epitélio está descamada, deixando somente as células da camada basal, que são descritas com uma aparência que lembra "carreira de pedras tumulares". No espaço vesicular são encontrados, com frequência, grupos de células que apresentam alterações degenerativas tais como tumefação dos núcleos, perda dos desmossomos, formato arredondado ou ovóide e hipercromatismo, sendo denominadas de células de Tzanck 4, 10, 21.

Estas são resultantes da acantólise celular em decorrência do edema pré-vesicular. No tecido conjuntivo, podem ser encontrados leucócitos polimorfonucleares e linfócitos em número variados. Ao contrário da maioria das doenças bolhosas, o pênfigo vulgar é caracterizado por escassez de infiltrado inflamatório no líquido da vesícula, exceto nos casos em que existe infecção secundária ${ }^{8,11,18 .}$.

Já na imunofluorescência direta do tecido perilesional revelará a presença de anticorpos lgG ou lgM e de componentes do complemento (normalmente C3) nos espaços intercelulares das células epiteliais, resultado que pode ser visto em quase todos pacientes ${ }^{17,22}$.

A imunofluorescência indireta é realizada com o soro do paciente portador de pênfigo, depois da reação deste com o tecido normal de controle, na tentativa de 9 demonstrar a presença de anticorpos circulantes. Essa técnica tem também sido utilizada no intuito de avaliar a gravidade da doença, que tem sido relacionada com a concentração de anticorpos circulantes. Assim, 
pode-se ajustar o esquema terapêuti$\mathrm{co}^{11,15}$.

$\bigcirc$ diagnóstico feito precocemente favorece o controle da doença. É necessário realizar diagnóstico diferencial com algumas doenças cujos sintomas lembram o pênfigo vulgar, além deste com as suas variantes. As principais lesões bolhosas/ulcerativas crônicas que fazem o diagnóstico diferencial com PV são: eritema multiforme, líquen plano (erosivo, bolhoso), penfigóide cicatricial, penfigóide bolhoso, liquen plano, pênfigo paraneoplásico e, menos frequentemente, ulcerações aftosas recorrente. Além dessas, existe uma condição genética rara conhecida como pênfigo benigno crônico ou doença de Hailey-Hailey, cuja manifestação se faz em forma de lesões cutâneas erosivas, no entanto o acometimento bucal não é comum 9,18

Os doentes que não apresentam melhora significativa com o uso de corticosteroides, podem ser tratadas com imunoglobulina e plasmaférese também podem ser indicadas as sulfonas sendo usadas nos quadros leves ou nas lesões mucosas resistentes de pênfigo vulgar ${ }^{8}$.

A sulfadiazina de prata é usada amplamente na terapia tópica de queimaduras, embora a dor devida à troca frequente de curativos seja comumente relatada. A hidrofibra Aquacel@ é curativo retentor e umidificador eficaz e seguro para o tratamento de queimaduras profundas. Após comprovação de sua eficácia, adicionou-se 1,2\% de prata, tendo sido criado o Aquacel $\mathrm{Ag}{ }^{\circledR}$, que assim adquiriu amplo espectro antimicrobiano ${ }^{21}$.

prognóstico dos pacientes com pênfigo vulgar continua reservado por causa das drogas utilizadas, seu potencial de efeitos colaterais e o tratamento a ser realizado ${ }^{11}$ pelo resto da vida. Antes do desenvolvimento da terapia com corticosteroides, de 60\% a $80 \%$ dos pacientes morriam, princi-

Os doentes que não apresentam melhora significativa com o uso de corticosteroides, podem ser tratadas com imunoglobulina e plasmaférese também podem ser indicadas as sulfonas sendo usadas nos quadros leves ou nas lesões mucosas resistentes de pênfigo vulgar

palmente devido a infecções e desequilíbrio hidroeletrolítico. Ainda assim, atualmente o índice de mortalidade associado com o pênfigo vulgar varia de cerca de $5 \%$ a $10 \%$, geralmente por causa das complicações com o uso em longo prazo dos corticosteroides sistêmicos ${ }^{16,19}$.

Sendo frequentemente o uso de cor- ticoides combinado com outros medicamentos imunossupressores lagentes que "restringem os esteroides"), como a azatioprina, além de antibióticos e antifúngicos para prevenção de infecções secundárias. Embora alguns clínicos utilizem corticosteroides tópicos no tratamento das lesões bucais sob a forma de enxaguatórios bucais e pomadas com ou sem veículo adesivo, a melhora observada é sem dúvida devida à absorção sistêmica dos agentes tópicos, o que resulta em uma dose sistêmica maior ${ }^{17}$.

Para pacientes com PF encontramos os seguintes diagnósticos baseados no livro de classificações e definições da Nanda (2013): integridade da pele prejudicada definida como, estado no qual o indivíduo está com o risco aumentado para ser invadido por organismos patogênicos; integridade tissular prejudicada por conta das lesões apresentadas em mucosas e tecido cutâneo ou subcutâneo; conforto alterado, relacionados aos ferimentos na pele e desconforto causados pela dor no local; distúrbio na imagem corporal e no auto conceito devido a confusão da imagem física, medo relacionado ao tratamento; risco de distúrbio no padrão do sono relacionado com desconforto causado pela patologia ${ }^{13}$.

$\bigcirc$ elemento semiotécnico para um diagnóstico mais simples do pênfigo vulgar é através do exame clínico com a verificação do sinal de Nikolsky. Esse sinal é considerado positivo quando, ao se friccionar fortemente a pele ou mucosa do paciente portador de pênfigo com a polpa digital ou com um instrumento rombo, ocorre o deslocamento do epitélio e surge no local uma superfície úmida tendendo para a cor rosada ou uma bolha. Embora este sinal seja de grande valia, não é patognomônico do pênfigo vulgar, podendo ser observado na grande maio- 
ria das lesões vesículobolhosas ${ }^{16,19,20}$

A biópsia é um elemento importante no diagnóstico e deve ser incluído tecido adjacente às lesões para realização de exame histopatológico e de imunofluorescência direta do tecido perilesional, fresco ou submetido à solução de Michel2, 6

Portanto o objetivo principal deste estudo foi descrever um estudo de caso de uma paciente com pênfigo vulgar internada em um hospital referência em doenças tropicais do Amazonas

\section{ESTUDO DE CASO}

Paciente G.A.S, 30 anos, indígena, com história de reinternação, acompanhada de seu esposo, deu entrada na unidade Mista da cidade de Anurí, interior do estado do Amazonas referindo dor no corpo, frio e apresentando erupções cutâneas generalizadas.

A mesma foi encaminhada para a Fundação de Medicina Tropical Dr. Heitor Vieira Dourado-FMT/HVD, em Manaus, para acompanhamento especializado. Paciente com história de conflitos familiar (relação conflituosa com uma das filhas) e parentes que fazem uso de drogas ilícitas. Grupo familiar extenso, composto por 09 (nove) pessoas, com renda de 01 (um) salário mínimo.

Paciente relata que há 10 dias iniciou quadro de bolhas finas que rapidamente evoluíram para erosões, com crostas hemáticas em região de face, dorso, tórax, abdome e membros inferiores-MMII (coxas e pernas). Relata ainda sangramento fácil e dor ao se encostar em objetos e ao deitar. Também refere prurido ao tomar banho. Encontra-se em posição sentada em cadeira próxima ao leito, em regular estado geral, relata não apresentar outras queixas e não encontra-se triste ou estressada. Paciente com sinais de baixa-alto-estima, chorosa, poliqueixosa, expressão facial de angustia, apresentando lesões bolhosas exsudativas, sangrantes aderentes as roupas de cama, disseminadas em todo o corpo, sem sinais de infecção, lesões de categoria dois.

No mesmo dia, foi solicitado acompanhamento e condutas da equipe de enfermagem por meio de parecer para a comissão de curativos da FMT/HVD.

Conduta da comissão de curativos:

- Analgesia 30 minutos antes do banho de aspersão;

- Banho de aspersão 1x ao dia com clorexidina a $2 \%$ antes do curativo;

- Limpeza com soro fisiologia 0,9\% ou água destilada lem temperatura ambiente),

- Junção de neomicina pomada (para profilaxia de bactérias da pele), + miconazol pomada (para profilaxia de fungos) + dexametazona pomada (para alivio do prurido)

- e cobertura da pele com ABD embebido em vaselina (para facilitar a remoção) e ataduras estéril (para fixar o ABD).

- Roupas pessoal (pijamas e etc) e de cama esterilizadas;

- Deixa-la em isolamento reverso (paciente com porta de entrada extensa e em uso de corticoides);

- Manter ferida longe de sujidades;

- Boa higiene corpórea e intima;

- Acionar a nutrição e dietética para adequação de dieta apropriada para pacientes com grandes perdas de tecidos;

- Acionar psicologia para acompanhamento do quadro de baixa autoestima.

\section{Imagens 1,2 e 3: primeiro dia do acompanhamento da enfermagem por meio da equipe da comissão de curativos}
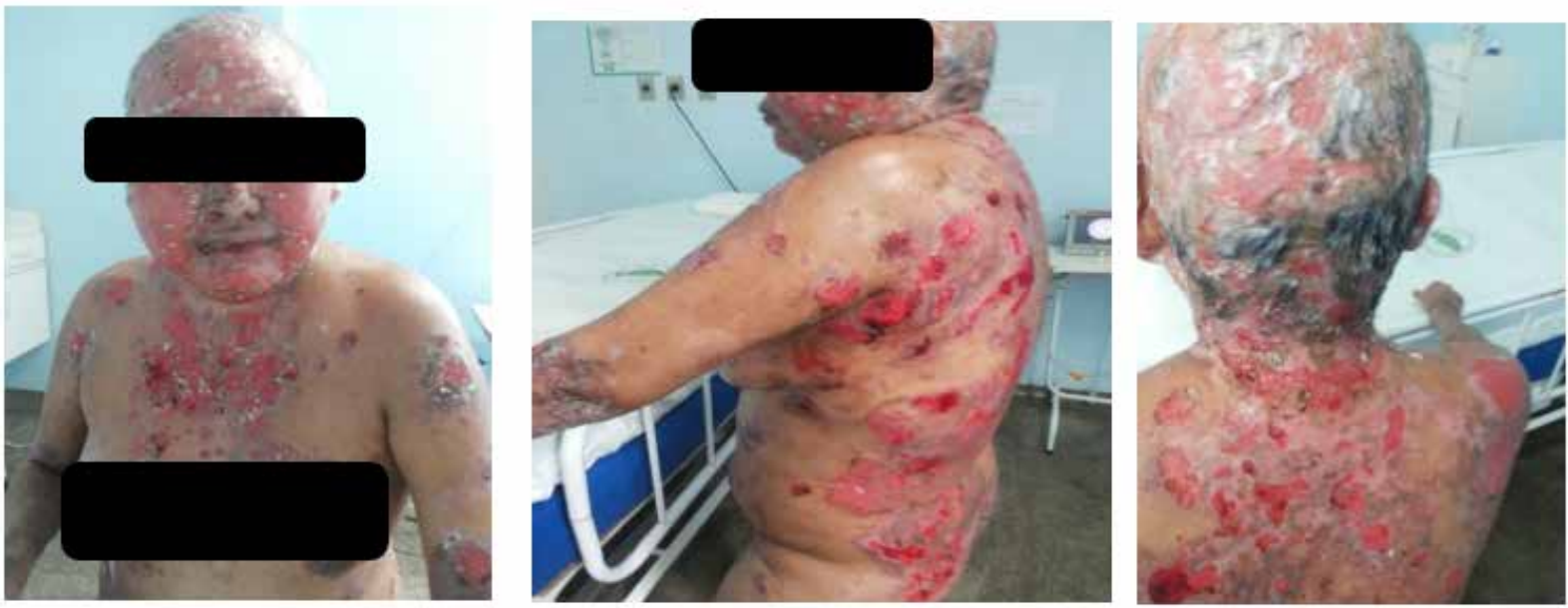


\section{DISCUSSÃO}

A vivência de cuidar de pessoas acometidas por doenças de pele como é o caso do pênfigo vulgar mostra a necessidade e a importância de cuidados especializados de enfermagem para a promoção do bem-estar/ conforto e manutenção da vida das mesmas. A complexidade do quadro apresentado por esses pacientes quando estão hospitalizados deve-se ao sofrimento pela dor, prognóstico grave, caráter desfigurante provocado pelas lesões extensas e suas repercussões nas esferas sociais e emocionais ${ }^{22}$.

Assim, para cuidar dessas pessoas de forma integral são imprescindíveis ao enfermeiro conhecimento e sensibilidade, não somente devido à especificidade dos cuidados, mas também o fato que torna o cuidar de enfermagem um verdadeiro desafio ${ }^{12,19}$

A dor nesses casos é típica, podendo ocorrer queixa de prurido e frio intenso pela perda de pele. Na maioria das vezes, as lesões exalam um odor fétido característico, o que leva à atração de insetos, podendo inclusive ocorrer infestação por miíase $e^{21,22}$.

Embora o indivíduo com essa patologia possa apresentar áreas extensas de pele sadia, ressalta-se que toda a epiderme está vulnerável a novas lesões. Assim, qualquer pressão na pele, até mesmo durante os cuidados de enfermagem rotineiros, pode aumentar a área lesada, sendo este fato constatado pelo sinal de Nikolski positivo $(+)$. Este sinal caracteriza-se pelo descolamento parcial ou total da epiderme, que se dá através da pressão com o dedo em pele perilesional ${ }^{18,20}$.

Diante da precariedade de enfermeiros especialistas na área de dermatologia, uma área ainda pouco explorada por esses profissionais, um dos desafios que se apresenta para o

Imagens 4 e 5: uma semana após o início do acompanhamento e confecção dos curativos pela equipe de enfermagem por meio da equipe da comissão de curativos'
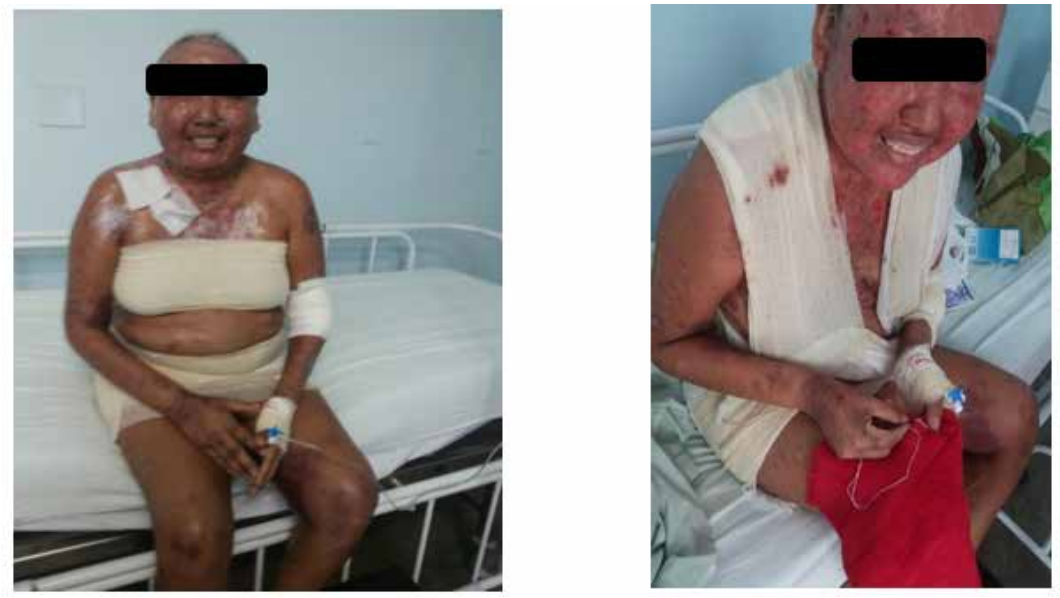

Imagens 6 e 7: paciente recebeu alta por melhora do quadro no $45^{\circ}$ dia de acompanhamento da equipe de enfermagem na confecção do curativo

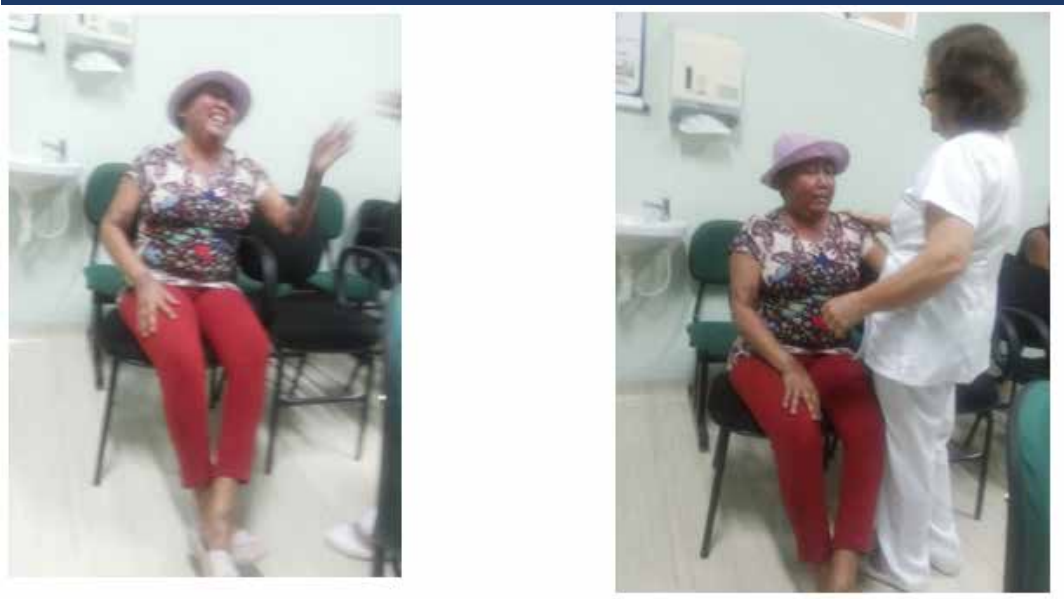

enfermeiro generalista é desenvolver um cuidado carente de conhecimentos específicos, que extrapolam as suas competências, levando-o a dilemas de ordem ética e moral no enfrentamento das exigências impostas ${ }^{22}$

Portanto, o desconhecimento da equipe de enfermagem, em especial o enfermeiro, em relação a esta clientela, pode provocar sensações de insegurança, medo e até repugnância durante o atendimento. As expressões e ações, fruto dessas sensações, provocam aumento do estigma e do sofri- mento, contrariando uma cultura voltada para a melhoria da qualidade do cuidado com vistas humanizadas ${ }^{13,16}$.

Indivíduos com pênfigo, independentemente de sua classificação, tendem a precisar de cuidados mais complexos, não só em ordem física, mas também emocionais e sociais. É de competência do enfermeiro proporcionar conforto e prevenção de agravos através dos conhecimentos adquiridos a partir das necessidades do seu cliente ${ }^{20}$

Os cuidados de enfermagem de- 
vem ser realizados correta e criteriosamente para que o período de remissão da doença seja o menor possível 11, 22.

Ha indicação do uso de lençóis de plástico estéreis para evitar aderências e assim facilitar a movimentação do cliente no leito, além da recomendação do uso de curativos oclusivos que devem ser hidratados para prevenir perda de eletrólitos e formação de crostas e evitar infecções e úlceras por pressão. $\bigcirc$ banho deve ser em água morna e, conforme a tolerância do paciente. Recomenda-se o uso de óleo como outra estratégia para evitar aderência a roupas ou lençóis 2, 6, 15 .

Em relação ao ambiente, este deve sempre estar limpo e, dependendo da gravidade da patologia o paciente deverá permanecer em isolamento reverso, ou seja, o paciente deve ficar protegido de infecções trazidas pelo servidor ${ }^{8,10}$.

A verificação dos sinais vitais e o acompanhamento da evolução das lesões e sinais flogísticos ao redor das lesões (dor, calor, rubor e edema) são importantes, assim como as informações sobre possíveis efeitos colaterais das medicações prescritas devem ser repassadas aos pacientes. Dessa maneira, o cuidado deve ser holístico e sempre incentivar o paciente ao autocuidado 8, 13, 19

Portanto, ter sob sua responsabilidade clientes acometidos por afecções cutaneomucosas, tais como é o caso do pênfigo vulgar, significa deparar-se com o despreparo da equipe, com a falta de ambiente adequado e do domínio da tecnologia do cuidado, prerrogativas estas que promovem uma assistência livre de riscos ${ }^{2,17}$.

\section{CONCLUSÃO}

$\bigcirc$ estudo em questão identifica toda a assistência de enfermagem prestada à uma paciente em convalescência por pênfigo, que foi internada em um hospital referência em doenças infectocontagiosas no Estado do
Amazonas e mostra uma alternativa de cuidado efetiva especialmente no que se refere à terapia tópica instituída de forma multiprofissional e interdisciplinar, fundamentando a prática do enfermeiro nesse cuidado. A assistência especializada de enfermagem no manejo e tratamento de lesões provenientes por pênfigo tem um papel fundamental e importante, pois proporciona questões do cuidado adequado à lesão, que passa pelas necessidades emocionais, nutricionais e de higiene, até chegar às questões relacionadas à exposição às vulnerabilidades sociais que condicionam sua situação de saúde e bem-estar do paciente. Com isso, concluiu-se que há uma grande necessidade de padronizações em cuidados relacionados as lesões de pele, principalmente as que exigem complexidade em seu manejo como os pacientes portadores de pênfigo vulgar e que o paciente deve ser assistido como um todo.

\section{Referências}

1. ALPSOY, E., AKMAN-KARAKAS, A., UZUNY, S. Geographic variations in epidemiology of two autoimmune bullous diseases: pemphigus and bullous pemphigoid. Arch Dermatol $\operatorname{Res}(2015)$ 307: 291. Acessado em: 02 de março de 2020. Disponível em: https://onlinelibrary.wiley.com/doi/ pdf/10.1111/ijd.14201.

2. AMORMINO S. A. F, BARBOSA A. A. M. Pênfigo vulgar: Revisão de Literatura e Relato de Caso Clínico. Acessado em: 19 de fevereiro de 2019. Visualizado em: R. Periodontia. [Internet] 2010; 20 (02). Disponível em: http://www. revistasobrape.com.br/arquivos/jun_2010/artigo6.pdf.

3. ATARZADEH, F. et al. A review on botanicals with wound healing activity for pemphigus vulgaris: perspective of traditional Persian medicine and conventional medicine. Avicena J Phytomed, v. 7, n. 6, p. 486-494, 2017. Acessado em: 02 de abril de 2020. Disponível em: https://www.ncbi.nlm. nih.gov/pmc/articles/PMC5745532/

4. BRANDÃO, E. S. et al. Proposta de reconhecimento de padrão de conforto em clientes com pênfigo vulgar utilizando a Lógica Fuzzy. Acessado em: 19 de fevereiro de 2019. Visualizado em: Rev Esc Enferm da USP, v. 47, n. 4, p. 958-964, 2013. Disponível em: http://www.scielo.br/pdf/ reeusp/v47n4/0080-6234-reeusp-47-4-0958.pdf.

5. GONÇALVES G. A. P, SALATHIEL A. M, ALVES D, BRITO M. M. C, FERRAZ T. S, ROSELINO A. M. F. Incidência do pênfigo vulgar ultrapassa a do pênfigo foliáceo em região endêmica para pênfigo foliáceo: análise de série histórica de 21 anos. Acessado em: 20 de fevereiro de 2019. Visualizado em: An Bras Dermatol. 2011 ; 86(6):1109-12. Disponível em: http://www.scielo.br/pdf/abd/v86n6/ v86n6a07.pdf

6. LEITE D. F. C, MACEDO M. P, SIMAS C. M. S, SOUZA L. C. D, LOPES F. F. Pênfigo vulgar na cavidade bucal: relato de caso clínico. Acessado em: 20 de fevereiro de 2019. Visualizado em: RFO, Passo Fundo, v. 20, n. 3, p. 367-371, set/dez. 2015. Disponível em: http://dx.doi. org/10.5335/rfo.v20i3.5272.

7. MIYAHARA, G.; CALLESTINI, R.; LAAWALL, M. A. Pênfigo vulgar: relato de caso clínico. UFES Rev. Odontol, vol. 6, n. 1, p. 24-29, 2004. Acessado em: 12 de abril de 2020. Disponível em:

8. https://www.researchgate.net/publication/Principais caracteristicas_do_Penfigo_Vulgar_Uma_revisao_de_litera- 
tura

9. MORAES, G.; GUERRA, M. Estudo Fisiopatológico do Pênfigo Vulgar. Revista Saúde em Foco, vol. 9, n. 3, p. 121128, 2017. Acessado em: 20 de fevereiro de 2020. Disponível em: http://portal.unisepe.com.br/unifia/wp- content/ uploads/sites/10001/2018/06/037_estudo.pdf.

10. NANDA. Diagnósticos de enfermagem da NANDA: definições e classificação (2012-2014). Porto Alegre, Artmed, 2013. Acessado em: 05 de março de 2020. Disponível em: https://enfermagemumarofissaodeamor.files.wordpress. com/2015/06/diagnc3b3stico-de-enfermagem-da-nanda-2012-2014.pdf.

1 1. OLIVEIRA A. P. R, LUZ E, NEGRELLO B. G, BURCI L. M, SOUZA S. J. P, PIEMONTE M. R. Atenção da enfermagem a pacientes com pênfigo vulgar e pênfigo foliáceo (fogo selvagem). Acessado em: 25 de fevereiro de 2019. Visualizado em: Revista Gestão \& Saúde, v. 15, n. 1, p. 27-33, 2016. Disponível em: http://www.herrero.com.br/files/revista/ fileOaOf857668893fb5587d5785a33fa 1 13.pdf.

12. REBOUÇAS, D.S; CERQUEIRA, L.S; COSTA, T.F; FERREIRA, T.G; NAVES, R.C; Zerbinati, L.P.S. Pênfigo vulgar, a importância do conhecimento do cirurgião dentista para um correto diagnóstico. Revista Bahiana de Odontologia. 2014 Dez; 5 (3): 174-181. Acessado em: 19 de novembro de 2019. Disponível em: https://www5.bahiana.edu.br/ index.php/odontologia/article/pfd.

13. RISSO, M..; VILLAPANDO, K.; PINHO, M.; FILHO, R. Pênfigo vulgar: relato de caso clínico. Rev. Gaúcha odontol., v. 59, n.3, p. 515-520, 2011 . Acessado em: 25 de novembro de 2019. Disponível em: http://www.revistargo.com. br/viewarticle. php?id=2707\&layout=abstract.

14. SALEH, M.A.; Salem H.; AZIZY H.E. Autoantibodies other than Anti-desmogleins in Pemphigus Vulgaris Patients. Indian J Dermatol. 2017 Jan-Feb; 62 (1): 47 - 51. Acessado em: 17 de novembro de 2019. Disponível em: https://onlinelibrary.wiley.com/doi/pdf/10.1111/ijd.14201.

15. SILVA, D.A.R. Diagnostico de enfermagem em pacientes com pênfigo foliáceo endêmico. Rev.Uningá, v. $17 \mathrm{n}$. 1, p. 19-26, 2014. Acessado em: 05 de março de 2020. Disponível em: http://revista.uninga.br/1487-13-4270-110-20180111.pdf.

16. SILVA, P. L. N. et al. Importância da comissão de curativos no tratamento das lesões cutâneas: um relato de experiência. REAS, Revista Eletrônica Acervo Saúde, 2017. Acessado em: 02 de abril de 2020. Disponível em: https:// www.acervosaude.com.br/doc/S-25_2017.pdf

17. VIEIRA, C. P. B. et al. Tecnologias utilizadas por enfermeiros no tratamento de feridas. Rev. Enferm UFPI. v. 6, n. 1, p. 65-70, 2017. Acessado em: 05 de abril de 2020. Disponível em: https://ojs.ufpi.br/index.php/reufpi /article/view/5852

18. WATTANAPLOY, S. et al. Randomized Controlled Trial of Polyhexanide/Betaine Gel Versus Silver Sulfadiazine for Partial-Thickness Burn Treatment .Int J Low Extrem Wounds., v. 16, n. 1, p. 45-50, 2017. Acessado em: 06 de abril de 2020. Disponível em: https://journals.sagepub.com/doi/full/10.1177/1534734617690949?url_ ver=Z39.88-2003\&rfr_id=ori:rid:crossref.org\&rfr_dat=cr_ pub\%3dpubmed

19. WIEGAND, C. et al. Effect of non-adhering dressings on promotion of fibroblast proliferation and wound healing in vitro. Sci Rep., v. 9, n. 1, 2019. Acessado em: 07 de abril de 2020. Disponível em: https://www.ncbi.nlm.nih.gov/ pmc/articles/PMC6416289/ 